\title{
ENDOMORPHISMS OF RIGHT IDEALS OF THE WEYL ALGEBRA
}

\section{J. T. STAFFORD}

\begin{abstract}
Let $A=A(k)$ be the first Weyl algebra over an infinite field $k$, let $P$ be any noncyclic, projective right ideal of $A$ and set $S=\operatorname{End}(P)$. We prove that, as $k$-algebras, $S \not A$. In contrast, there exists a noncyclic, projective right ideal $Q$ of $S$ such that $S \cong \operatorname{End}(Q)$. Thus, despite the fact that they are Morita equivalent, $S$ and $A$ have surprisingly different properties. For example, under the canonical maps, $\operatorname{Aut}_{k}(A) \cong \operatorname{Pic}_{k}(A) \cong \operatorname{Pic}_{k}(S)$. In contrast, $\operatorname{Aut}_{k}(S)$ has infinite index in $\operatorname{Pic}_{k}(S)$.
\end{abstract}

Introduction. Given a commutative domain $R$, the (first) Weyl algebra $A(R)$ is defined to be the associative $R$-algebra (thus $R$ is central subring) generated by elements $x$ and $y$ subject to the relation $x y-y x=1$. When no ambiguity is possible, we write $A$ for $A(R)$.

Let $F$ be a field of characteristic zero. Then $A=A(F)$ is a simple ring and, indeed, may be thought of as one of the nicest and most important examples of simple Noetherian rings. The initial motivation for this paper was the following result of Smith. If $P$ is the noncyclic right ideal $P=x^{2} A+(x y+1) A$ of $A$ then $\operatorname{End}(P) \not A$ (as $F$-algebras) [11]. Note that, as $\operatorname{char}(F)=0, A$ is a simple hereditary ring and so $\operatorname{End}(P)$ is automatically Morita equivalent to $A$. Even worse, as $P \oplus P \cong A \oplus A[\mathbf{1 7}]$, the full matrix rings $M_{2}(A)$ and $M_{2}(\operatorname{End}(P))$ are isomorphic. Thus any proof of Smith's result must be fairly subtle and may therefore provide useful invariants for $A$.

The first main aim of this paper is to generalize Smith's result to an arbitrary projective right ideal of $A$. While the proof of this is harder than that of Smith's result it does provide a more informative proof in the sense that, unlike Smith, we do not (and cannot) require an explicit description of $\operatorname{End}(P)$. For the rest of this introduction $k$ will denote a field of arbitrary characteristic and all isomorphisms of rings will be $k$-algebra isomorphisms.

THEOREM A. Let $P$ be a projective right ideal of $A=A(k)$. Then $\operatorname{End}(P) \cong A$ if and only if $P$ is a cyclic right ideal of $A$. (See Theorem 3.1.)

This has some easy corollaries:

COROLlaRY B. Let $P$ and $Q$ be projective right ideals of $A=A(k)$. Then $\operatorname{End}(P) \cong \operatorname{End}(Q)$ if and only if $P=t \sigma(Q)$ for some $t \in D(k)$, the division ring of fractions of $A$, and $\sigma \in \operatorname{Aut}_{k}(A)$, the group of $k$-automorphisms of $A$.

Received by the editors February 13, 1986.

1980 Mathematics Subject Classification (1985 Revision). Primary 16A65, 16A72, 16A19.

Supported in part by an NSF grant. 
Secondly, if $F$ is a field of characteristic zero, $A(F)$ can be thought of as the ring of differential operators on the affine line $\mathrm{A}$ over $F$. Theorem $\mathrm{A}$ immediately gives information on the ring of differential operators $D(X)$ on a more general curve $X$.

COROLlaRY C. Let $X$ be a singular, irreducible, affine, algebraic curve with normalization $\mathrm{A}$. Then $D(X) \nRightarrow D(\mathrm{~A})=A(F)$. (However $D(X)$ is frequently Morita equivalent to $D(A)-$ see $\S 5$ for the details.)

While Corollary B contains Theorem $\mathrm{A}$ as a special case, it is in one sense a much weaker result, in as much as one needs to involve Aut $(A)$. Curiously, for any noncyclic $Q$, Corollary B fails if one demands that $\sigma=1$. Remarkably, this in turn implies that Theorem A becomes false if we replace $A(k)$ by $\operatorname{End} Q$.

ThEOREM D. Let $k$ be an infinite field. Let $R$ be a domain that is Morita equivalent, but not isomorphic to $A(k)$. Then there exists a noncyclic, projective right ideal $P$ of $R$ such that $R \cong \operatorname{End}(P)$. (See Theorem 4.3.)

Theorems A and D suggest that there should be natural invariants to distinguish between $A(k)$ and $R$. One such invariant is provided by an appropriate factor of the Picard group, $\mathrm{Pic}_{k}(-)$ (see $\S 4$ for the definition).

COROLlary E. Let $k$ be an infinite field and $R$ a domain that is Morita equivalent to $A=A(k)$. Then there is a natural embedding $\omega: \operatorname{Aut}_{k}(R) \rightarrow \operatorname{Pic}_{k}(R)$. If $R \cong A$ then $\omega$ is an isomorphism, but if $R \not A$ then the coset space $\operatorname{Pic}_{k}(R) / \operatorname{Im}(\omega)$ is infinite. (See Theorem 4.7.)

Corollary $\mathrm{E}$ (and Theorem $\mathrm{D}$ ) are surprising in that $A$ and $R$ are such similar rings; one even has $M_{n}(A) \cong M_{n}(R)$ for $n \geq 3$. In particular, since they are Morita equivalent, $\operatorname{Pic}_{k}(A) \cong \operatorname{Pic}_{k}(R)$. This suggests that $\operatorname{Pic}_{k}(S) / \operatorname{Im}(\omega)$ and possibly $\operatorname{Pic}_{k}(S)$, can provide sensitive invariants for an arbitrary simple Noetherian ring $S$. Of course, the proofs of this paper also suggest that they will be very difficult to calculate.

The proof of Theorem A falls naturally into two halves. One first considers the case when char $k=p>0$. In this case $A=A(k)$ is a finitely generated module over its center $Z(A)=k\left[x^{p}, y^{p}\right]$. Here one proves first that (apart from a few exceptions) every $k$-automorphism of $Z(A)$ extends to an automorphism of $A$. Theorem $\mathrm{A}$ is then an easy consequence of the Skolem-Noether theorem (see $\S 2$ ). For fields of characteristic zero, the theorem is proven by a reduction to characteristic $p>0$ (see $\S 3$ ). We should remark that this reduction is fairly formal and will hold in considerably greater generality than just the ring $A$. Thus the crux of the argument is really to show that, in positive characteristic, one can extend $k$-automorphisms from $Z(A)$ to $A$. In particular, the dichotomy presented by Theorem $\mathrm{D}$ implies that in positive characteristic one cannot extend all automorphisms from $Z(R)$ to $R$. (The precise statement of this result needs a little more care-see Corollary 4.8 and compare with Theorem 2.4.)

The idea of using a 'reduction $\bmod p$ ' to prove Theorem A was suggested by an unpublished result of Makar-Limanov and Schofield who used the same method to find an example of a division ring $E$ that is finite dimensional over $D(k)$, but not isomorphic to $D(k)$. I am grateful to them for sharing this result with me. Another recent application of this method can be found in [5]. 
There is an intriguing analogy between the results of this paper and the following theorem of Paramila. Let $E$ be a division ring that is $p^{2}$-dimensional over its center $k$, for some prime $p$, and set $S=E\left[x_{1}, \ldots, x_{n}\right]$ for the polynomial extension of $E$ in $n \geq 2$ commuting indeterminates. Then there exist noncyclic, projective right ideals in $S$ and, for any such right ideal, say $P$, one has $\operatorname{End}(P) \not S$. This is because the only units in $\operatorname{End}(P)$ are the nonzero elements of $k$. This suggests the following curious conjecture. Let $S$ be one of the rings for which $[\mathbf{1 4}]$ provides noncyclic, projective right ideals. (Such rings include group rings of poly (infinite cyclic) groups and enveloping algebras of finite dimensional Lie algebras, as well as $A(k)$ and $E\left[x_{1}, \ldots, x_{n}\right]$.) Then $\operatorname{End}(P) \neq S$ for any noncyclic, projective right ideal $P$ of $S$. The results mentioned earlier suggest that this nonisomorphism would be caused by the fact that $\operatorname{Aut}_{k}($ End $P)$ is $\operatorname{smaller}$ than $\operatorname{Aut}_{k}(S)$.

1. Generalities. In this section we collect together various well-known results about the Weyl algebra that we will need. Many of these observations will be used without comment in the body of the paper. Throughout the paper, $k$ will denote a field and all homomorphisms between $k$-algebras will be $k$-algebra homomorphisms.

Observe first that $A=A(k)$ is a Noetherian domain and so by Goldie's Theorem it has a division ring of fractions, which will be denoted by $D(k)$. Fix a finitely generated, nonzero right $A$-submodule $M$ of $D=D(k)$. Then we will always identify $\operatorname{End}(M)$ with $O_{l}(M)=\{f \in D: f M \subseteq M\}$. We remark that, whatever the characteristic of $k, A$ is maximal order in $D$; that is, there exists no ring $A \varsubsetneqq S \subset D$ such that $a S b \subseteq A$ for some $a, b \in D \backslash\{0\}$ [2, Example 3.2(a)]. If $M$ is also a left module over a second order $R$ in $D$ then this implies that

$$
\text { if } T=\operatorname{End}\left({ }_{R} M\right), \quad \text { then } T=A \text {. }
$$

To see this note that we may again identify $T$ with a subalgebra of $D$. Pick $s \in D \backslash\{0\}$ such that $s M \subseteq A$. Then for any $m \in M \backslash\{0\}$ we have $s m T \subseteq s M \subseteq A$. Thus $T=A$ by the maximality of $A$. Of course, (1.1) holds with any maximal order in place of $A$.

Suppose, from now on, that $M$ is also projective as a right $A$-module. Then End $M$ is also a maximal order [2, Proposition 1.3]. We will also identify $\operatorname{Hom}(M, A)$ with $M^{*}=\{f \in D: f M \subseteq A\}$. Note that, by the Dual Basis Lemma, $\operatorname{End}(M)=$ $M M^{*}$. This also means that there is no ambiguity in the notation $M^{*}$, in the sense that

$$
M^{*}=\{f \in D: M f \subseteq \operatorname{End} P\} \cong \operatorname{Hom}_{\operatorname{End}(M)}(M, \operatorname{End}(M)) .
$$

Similarly, if $\sigma \in \operatorname{Aut}_{k}(D)$ then $\sigma(M)$ is a right $\sigma(A)$-module, and hence

$$
\sigma\left(M^{*}\right)=\{g \in D: g \sigma(M) \subseteq \sigma(M)\}=[\sigma(M)]^{*} .
$$

We now turn to a consideration of projective right ideals of $A$. One of the main techniques in this paper is to change our base field (or ring) and we therefore need a method for ensuring that noncyclic right ideals remain noncyclic under such changes. The most effective method for checking this seems to be to use the standard filtration. Let $R$ be any commutative Noetherian domain and pick $f \in A(R) \backslash\{0\}$. Then $f$ may be uniquely written as $f=\sum_{0}^{m} x^{i} f_{i}$ for some $m$ and $f_{i} \in R[y]$. If $f_{m} \neq 0$ then we define:

$$
\operatorname{deg}(f)=m \text { and } L(f)=f_{m}, \quad \text { the leading coefficient of } f .
$$


Note that, for any $f, g \in A(R) \backslash\{0\}$ we have

$$
\operatorname{deg}(f g)=\operatorname{deg} f+\operatorname{deg} g \quad \text { and } \quad L(f g)=L(f) L(g)
$$

Similarly, if $I$ is a nonzero right ideal of $A(R)$ we define, for $n \geq 0$,

$$
L_{n}(I)=\{L(f): f \in I \text { with } \operatorname{deg} f=n\} .
$$

Observe that each $L_{n}(I)$ is an ideal of $R[y]$ and $L_{n}(I) \subseteq L_{n+1}(I)$ for all $n$. Since $R$ is Noetherian this implies that $\bigcup L_{i}(I)=L_{n}(I)$ for some $n \geq 0$, and we denote this ideal by $L_{\infty}(I)$. We also write $L_{\min }(I)$ for $L_{r}(I)$ where $r$ is the minimal $i$ such that $L_{i}(I) \neq 0$. The following observation is useful for testing whether $I$ is cyclic.

$I$ is cyclic if and only if $L_{\min }(I)$ is a cyclic ideal of $R[y]$ and $L_{n}(I)=$ $L_{n+1}(I)$ for all $n$ such that $L_{n}(I) \neq 0$.

2. Endomorphism rings in characteristic $p$. In this section $k$ will always denote a field of characteristic $p>0$. The main aim of this section is to prove Theorem $\mathrm{A}$ of the introduction for $A(k)$ and this will be an easy consequence of the fact that we can lift (most) $k$-automorphisms of the center $Z(A)$ of $A=A(k)$ to automorphisms of $A$. By $[\mathbf{9}], Z(A)=k\left[x^{p}, y^{p}\right]$ is a polynomial ring and so by $[\mathbf{1 6}]$ the automorphisms of $Z(A)$ are known. We begin by rephrasing Van der Kulk's result in a form that is appropriate to our needs.

LEMMA 2.1. Let $k\left[z_{0}, z_{1}\right]$ be a polynomial ring in two variables over $k$ and define $k$-automorphisms $\sigma_{i f}$ by $\sigma_{i f}\left(z_{i}\right)=z_{i}+f$ and $\sigma_{i f}\left(z_{1-i}\right)=z_{1-i}$, where $f \in$ $k\left[z_{1-i}\right]$ and $i=0,1$. Let $G$ be the subgroup of Aut $\left(k\left[z_{0}, z_{1}\right]\right)$ generated by the $\sigma_{i f}$. Let $H$ be the subgroup $\operatorname{Aut}\left(k\left[z_{0}, z_{1}\right]\right)$ consisting of the scalar maps $\tau_{\lambda}$ defined by $\tau_{\lambda}\left(z_{0}\right)=z_{0}$ and $\tau_{\lambda}\left(z_{1}\right)=\lambda z_{1}$ for some $\lambda \in k \backslash\{0\}$. Then any $\theta \in$ Aut $k\left[z_{0}, z_{1}\right]$ can be written $\theta=h g$ for some $h \in H$ and $g \in G$.

Proof. By [16, Theorem 1], Aut $\left(k\left[z_{0}, z_{1}\right]\right)$ is generated by $G$ and automorphisms $\phi$ of the form $\phi\left(z_{0}\right)=a z_{0}+b z_{1}+c$ and $\phi\left(z_{1}\right)=d z_{0}+e z_{1}+f$ where $a, \ldots, f \in k$ and $\operatorname{det}\left(\begin{array}{ll}a & b \\ d & e\end{array}\right) \neq 0$. It is an easy exercise to show that $\phi=\phi_{1} \phi_{2}$ for some $\phi_{1} \in H$ and $\phi_{2} \in G$. Thus $\operatorname{Aut}\left(k\left[z_{0}, z_{1}\right]\right)$ is generated by $G$ and $H$, from which it follows easily that $G$ is a normal subgroup, as required.

We will continue to write $G$ and $H$ for the subgroups of Aut $Z(A)$ defined by Lemma 2.1 (under the identification, say, $z_{0}=x^{p}$ and $z_{1}=y^{p}$ ). We now consider which automorphisms of $Z(A)$ extend to $A$. For the subgroup $G$ this is easy.

LEMMA 2.2. Suppose that $k$ is a perfect field. Then any $g \in G$ extends to an automorphism of $A=A(k)$. 1].

REMARK. A proof of this lemma is also implicit within the proof of $[\mathbf{5}$, Theorem

PROOF. It clearly suffices to prove the lemma for $\sigma=\sigma_{0 f}$; the case of $\sigma_{1 g}$ being symmetric. Thus $\sigma\left(x^{p}\right)=x^{p}+f$ and $\sigma\left(y^{p}\right)=y^{p}$, where $f=\sum_{0}^{m} f_{i} y^{p i} \in k\left[y^{p}\right]$. We prove the result by induction on $m$. As $k$ is perfect, we may write $f_{m}=g_{m}^{p}$ for some $g_{m} \in k$ and hence define a $k$-automorphism $\tau$ on $A$ by $\tau(x)=x+g_{m} y^{m}$ and $\tau(y)=y$. (Note that, if $m=0$, then $\left.\tau\right|_{Z(A)}=\sigma$ and so the induction does start.) Since $\tau(x)$ and $y=\tau(y)$ are generators of $A$ that satisfy $\tau(x) y-y \tau(x)=1$, we 
necessarily have $\tau(x)^{p} \in Z(A)=k\left[x^{p}, y^{p}\right]$. The obvious degree argument therefore forces $\tau\left(x^{p}\right)$ to have the form

$$
\tau\left(x^{p}\right)=x^{p}+g_{m}^{p} y^{m p}+\sum_{0}^{m-1} h_{i} y^{p i}, \quad \text { for some } h_{i} \in k .
$$

By induction, there exists $\tau_{1} \in \operatorname{Aut}(A)$ such that $\tau_{1}\left(x^{p}\right)=x^{p}+\sum_{0}^{m-1}\left(f_{i}-h_{i}\right) y^{p i}$ and $\tau_{1}\left(y^{p}\right)=y^{p}$. But now

$$
\begin{aligned}
\tau \tau_{1}\left(x^{p}\right) & =\tau\left(x^{p}+\sum\left(f_{i}-h_{i}\right) y^{p i}\right)=x^{p}+g_{m}^{p} y^{m p}+\sum_{0}^{m-1} f_{i} y^{p i} \\
& =\sigma\left(x^{p}\right) .
\end{aligned}
$$

Thus $\sigma$ does indeed extend to an automorphism of $A$.

It is worth remarking that the extension of $\sigma \in \operatorname{Aut} Z(A)$ to an automorphism $\tau$ of $A$ defined by Lemma 2.2 may be a little involved. For example, if char $k=2$ and $\sigma=\sigma_{0, y^{2}}$, then $\tau$ is defined by $\tau(x)=x+y+1$, rather than by $\tau(x)=x+y$.

We now turn to elements of the subgroup $H$. While (apart from the identity element) no $h \in H$ extends to an automorphism of $A$, as we next show, $h$ also does not extend to an automorphism of $D(k)$. As such, $h$ will not arise in our problem about endomorphism rings.

LEMMA 2.3. Let $k$ be any field (of characteristic $p$ ) and $\sigma \in H$. Then the following are equivalent:

(i) $\sigma=1$,

(ii) $\sigma$ extends to an automorphism of $A(k)$,

(iii) $\sigma$ extends to an automorphism of $D(k)$.

ProOF. The implications (i) $\Rightarrow($ ii $) \Rightarrow($ iii) are trivial and so it remains to prove (iii) $\Rightarrow$ (i). Let $\sigma$ be defined by $\sigma\left(x^{p}\right)=x^{p}$ and $\sigma\left(y^{p}\right)=\lambda y^{p}$ for some $\lambda \in k \backslash\{0\}$ and suppose that $\sigma$ extends to an automorphism $\tau$ of $D(k)$ defined, say, by $\tau(x)=f$ and $\tau(y)=g$. In order to study these elements it is convenient to embed $D(k)$ in the ring of inverse power series:

$$
D(k) \subset T=\left\{\sum_{-\infty}^{n} x^{i} t_{i}: t_{i} \in k(y), n \in \mathbb{Z}\right\} .
$$

Given $t=\sum_{-\infty}^{n} x^{i} t_{i} \in T$ with $t_{n} \neq 0$, we define $\operatorname{deg} t=n$ and $L(t)=t_{n}$, the leading coefficient of $t$. The observations given in (1.4) have analogues in the present situation. Thus, given $t, s \in T \backslash\{0\}$, then

$$
\operatorname{deg}(t s)=\operatorname{deg} t+\operatorname{deg} s \text { and } L(t s)=L(t) L(s) .
$$

For these and further results about $T$ the reader is referred to [4], particularly $\S 3$.

Now write $f=\sum_{-\infty}^{m} x^{i} f_{i}$ and $g=\sum_{-\infty}^{n} x^{i} g_{i}$ for appropriate $f_{i}$ and $g_{j} \in k(y)$. Since $f^{p}=\tau\left(x^{p}\right)=x^{p}$, the comments of the last paragraph imply that $\operatorname{deg}(f)=1$ with $L(f)^{p}=1$, whence $L(f)=1$. Similarly, $\operatorname{deg}(g)=0$ with $L(g)=\mu$ for some $\mu \in k$ such that $\mu^{p}=\lambda$. Now for any $i$ we have $\left[x, x^{i} g_{i}\right]=x^{i}\left(\partial\left(g_{i}\right) / \partial y\right)$ and 
$\left[x^{i} f_{i}, y\right]=i x^{i-1} f_{i}$. Thus

$$
\begin{aligned}
1=[f, g] & =\left[x+\sum_{-\infty}^{0} x^{i} f_{i}, \mu y+\sum_{-\infty}^{-1} x^{i} g_{i}\right] \\
& =[x, \mu y]+\text { terms of degree }<0 \\
& =\mu+\text { terms of degree }<0 .
\end{aligned}
$$

Thus $\mu=1$ and $\sigma=1$; as required.

THEOREM 2.4. Let $k$ be a perfect field of characteristic $p>0$ and put $A=$ $A(k)$. Suppose that $\sigma \in \operatorname{Aut}(Z(A))$. Then the following are equivalent:

(i) $\sigma \in G$,

(ii) $\sigma$ extends to an automorphism of $A$,

(iii) $\sigma$ extends to an automorphism of $D(k)$.

PROOF. By Lemma 2.1 we may write $\sigma=h g$ for some $h \in H$ and $g \in G$. By Lemma 2.2, $g$ extends to an automorphism of $A$ and hence to one of $D(k)$. Thus if (iii) holds then $h=\sigma g^{-1}$ also extends to an automorphism of $D(k)$. Hence, by Lemma 2.3, $h=1$ and $\sigma=g \in G$. That (i) implies (ii) is just Lemma 2.2 and (ii) $\Rightarrow$ (iii) is automatic.

REMARK 2.5. It would be interesting to know if one could find a more intuitive proof of Theorem 2.4, as this may allow one to extend the theorem to other rings (for example to $A_{n}=A \otimes_{k} \cdots \otimes_{k} A$ ). As the remaining steps in the proof of Theorem A hold in considerable generality, this would allow one to extend Theorem A to these more general rings. However, some subtleties must remain in any proof of Theorem 2.4 since, as will be shown in $\S 4$, the result fails for any domain Morita equivalent, but not isomorphic to $A(k)$.

The first case of Theorem A now follows easily.

PROPOSITION 2.6. Let $k$ be a perfect field of characteristic $p>0$, and suppose that $P$ is a noncyclic, projective right ideal of $A=A(k)$. Then $\operatorname{End}(P) \not A$.

Proof. Suppose that $\sigma$ is the given isomorphism from $S=\operatorname{End}(P)$ to $A$. If $Z=Z(A)$ then $Z P=P Z \subseteq P$ and so $Z \subset S$. Since $Z \subset Z(D(k))$ we see that $Z \subseteq Z(S)$ and, by a symmetric argument, $Z=Z(S)$. Thus $\sigma$ restricts to an automorphism, say $\tau$, of $Z$. Also, as $D(k)$ is the division ring of fractions $S, \sigma$ (and $\tau$ ) extend to an automorphism of $D(k)$. Thus, by Theorem 2.4, $\tau$ extends to an automorphism $\theta$ of $A$. Therefore, replacing $\sigma$ by $\theta^{-1} \sigma$ we may assume that $\sigma$ is a $Z$ isomorphism. Thus by the Skolem-Noether theorem, $\sigma$ is an inner automorphism of $D(k)$; say $\sigma(a)=t a t^{-1}$ for some $t \in D$. Since $A$ is a PI ring, there exists $v \in Z \backslash\{0\}$ such that $t v \in A$. Of course we may replace $t$ by $t v$. Now, $S=t^{-1} A t$ and so $\operatorname{End}(t P)=t(\operatorname{End} P) t^{-1}=A$. But as $t P \subseteq A$ this just says that $t P$ is a two-sided ideal of $A$. Since $A$ is an Azumaya algebra, [9, Théorème 2], this implies that $t P=I A \cong I \otimes_{Z} A$ for some ideal $I$ of $Z$. But as $A$ is a free $Z$-module, the proof of $[\mathbf{1 0}$, Theorem 9.39] implies that $I$ is a projective ideal of the UFD $Z=k\left[x^{p}, y^{p}\right]$. Thus $I$, and hence $P$ are cyclic; as required.

It is easy to remove the hypothesis that $k$ is perfect in Proposition 2.6.

COROLLARY 2.7. Let $k$ be any field of characteristic $p>0$ and suppose that $P$ is a projective noncyclic right ideal of $A(k)$. Then $\operatorname{End}(P) \not A(k)$. 
PROOF. Let $\bar{k}$ be the algebraic closure of $k$. If $\sigma$ is an isomorphism from $A(k)$ to $\operatorname{End}(P)$, then certainly $\sigma$ extends to an isomorphism

$$
\bar{\sigma}: A(\bar{k}) \cong A(k) \otimes_{k} \bar{k} \rightarrow \operatorname{End}(P) \otimes_{k} \bar{k} \cong \operatorname{End}\left(P \otimes_{A(k)} A(\bar{k})\right) .
$$

Thus, by Corollary 2.6 it remains to prove that $P \bar{k}=P \otimes A(\bar{k})$ is not cyclic as an $A(\bar{k})$-module. To do this we use the notation of $\S 1$. As $P$ is not cyclic, (1.5) implies that $L_{i}(P) \neq L_{j}(P)$ for some $i<j$ for which $L_{i}(P) \neq 0$. Since each $L_{n}(P)$ is a cyclic ideal of $k[y]$ it is easy to see that $L_{n}(P \bar{k})=L_{n}(P) \otimes_{k} \bar{k}$ (where $L_{n}(P \bar{k})$ is defined by considering $P \bar{k}$ as a right ideal of $A(\bar{k}))$. Thus $L_{i}(P \bar{k}) \neq L_{j}(P \bar{k})$ and $P \bar{k}$ is not cyclic. By Proposition 2.6 this gives the required contradiction.

REMARK. If $S=\mathbb{R}[x, y, z] /\left(x^{2}+y^{2}+z^{2}-1\right)$ is the coordinate ring of the 2sphere, then there exists a nonfree, projective $S$-module $P$ such that $P \otimes_{\mathbf{R}} \mathbb{C}$ is free $\left[15\right.$, p. 269]. As rank $P=2, P \otimes_{S}\left(M_{2}(S)\right)$ is therefore isomorphic to a noncyclic, projective right ideal $\bar{P}$ of $\bar{S}=M_{2}(S)$ for which $\bar{P} \otimes_{\mathbf{R}} \mathbb{C}$ is cyclic. Thus the proof of Corollary 2.7 does not hold for an arbitrary $k$-algebra.

In [3, Théorème 8.10], Dixmier gives generators for $\operatorname{Aut}_{F}(A(F))$ when $\operatorname{char}(F)=$ 0 . The following analogue of Dixmier's result for positive characteristic is implicit in the proof of Theorem 2.4.

COROLlaRY 2.8. Let $k$ be a perfect field. Then $\operatorname{Aut}_{k}(A(k))$ is generated by automorphisms $\sigma_{\text {if }}$ that are defined as follows:

$$
\sigma_{1 f}(x)=x+f, \quad \sigma_{1 f}(y)=y, \quad \text { where } f \in k[y]
$$

and

$$
\sigma_{2 f}(x)=x, \quad \sigma_{2 f}(y)=y+f, \quad \text { where } f \in k[x] .
$$

A similar (and independent) proof of Corollary 2.8 over an arbitrary field of positive characteristic is given in [5, Theorem $\mathbf{1}]$.

3. Endomorphisms in characteristic zero. In this section we complete the proof of Theorem A of the introduction and give some of its easier consequences, among them Corollary B of the introduction. The basic idea is to reduce $\bmod p$ by replacing the base field $k$ first by a finitely generated $\mathbb{Z}$-algebra $R$ and then by a finite factor field of $R$.

THEOREM 3.1. Let $k$ be a field (of any characteristic) and $P$ a projective right ideal of $A=A(k)$. Then $\operatorname{End}(P) \cong A$ if and only if $P$ is cyclic.

Proof. Clearly, if $P$ is cyclic then $\operatorname{End}(P) \cong A$. Thus we may assume that $P$ is not cyclic and, by way of contradiction, that $\operatorname{End}(P) \cong A$. By Corollary 2.7 we may assume that $\operatorname{char}(k)=0$.

Step 1 . Replace $k$ by a finitely generated $\mathbb{Z}$-algebra $R$.

Informally speaking, all our hypotheses can be phrased in terms of a finite number of elements, and so we may replace $k$ by the $\mathbb{Z}$-algebra generated by the coefficients of these elements.

More formally, pick a module $N$ and an integer $r$ such that $P \oplus N \cong A^{(r)}$. Thus $\operatorname{End}\left(A^{(r)}\right)=M_{r}(A)$ and for some idempotent $e \in M_{r}(A)$ we may identity $P$ with $e A^{(r)}$, in which case End $P=e M_{r}(A) e$. We now have an isomorphism $\sigma: A \rightarrow$ $e M_{r}(A) e$. Pick a finitely generated $\mathbb{Z}$-subalgebra $R$ of $k$ such that (i) $e \in M_{r}(A(R))$, considered as a $\mathbb{Z}$-subalgebra of $M_{r}(A(k))$, (ii) $\sigma(x)$ and $\sigma(y) \in e M_{r}(A(R)) e$ and 
(iii) $\sigma^{-1}(e f e) \in A(R)$ where $f$ runs through the canonical generators $e_{i j} x$ and $e_{i j} y$ of $M_{r}(A(R))$ as an $R$-algebra. We claim this algebra $R$ satisfies our requirements.

Note that $A(R)$ is automatically a Noetherian domain. First, as $e \in M_{r}(A(R))$ we have

$$
e A(R)^{(r)} \subseteq Q=A(R)^{(r)} \cap P \quad \text { and } \quad(1-e) A(R)^{(r)} \subseteq A(R)^{(r)} \cap N=N_{1} .
$$

Since $Q \cap N_{1} \subseteq P \cap N=0$, this implies that $A(R)^{(r)}=Q \oplus N_{1}$ and $Q=e A(R)^{(r)}$. Thus $Q$ is projective. Further, since

$$
Q \otimes_{R} k=e A(R)^{(r)} \otimes_{R} k=e A(k)^{(r)}=P,
$$

$Q$ cannot be cyclic as an $A(R)$-module. A similar argument shows that $Q$ has uniform dimension one; i.e., that $Q$ is isomorphic to a right ideal of $A(R)$. Finally as $Q=e A(R)^{(r)}, \operatorname{End}_{A(R)}(Q) \cong e M_{r}(A(R)) e$ and so by parts (ii) and (iii), $\sigma$ and $\sigma^{-1}$ restrict to $R$-algebra homomorphisms between $A(R)$ and $e M_{r}(A(R)) e$. Since $\sigma \sigma^{-1}=$ Id and $\sigma^{-1} \sigma=$ Id they are necessarily isomorphisms; as required.

Step 2. Replace $R$ by a finite factor field.

To avoid some notation we will write $A$ for $A(R)$. Note first that we may always replace $R$ by $R\left[c^{-1}\right]$ for $c \in R \backslash\{0\}$ without changing our hypotheses, simply because the only requirement on $R$ in Step 1 was that it should contain certain elements.

Let $M$ be a maximal ideal of $R$. As $Q$ is a projective right ideal of $A$, certainly $Q / Q M$ is a projective $A / M A$-module. Since $Q$ is a projective module over the commutative domain $R$, certainly $Q / Q M \neq 0$. But we also have an $R / M$-algebra homomorphism

$$
\phi: A(R / M) \cong A / M A \stackrel{\sim}{\rightarrow} \operatorname{End} Q / M \text { End } Q \rightarrow \operatorname{End}(Q / Q M)
$$

where the final map is surjective by the projectivity of $Q$. But $A(R / M)$ is a domain and $Q / Q M$ is a nonzero projective $A(R / M)$-module. Thus $\phi$ cannot have a kernel and so $A(R / M) \cong \operatorname{End}(Q / Q M)$. Of course, since $\operatorname{End}(Q / Q M)$ is therefore a. domain, $Q / Q M$ is a uniform $A(R / M)$-module and hence isomorphic to a right ideal of $A(R / M)$. Also, $R / M$ is a finite field. Thus if $Q / Q M$ remains noncyclic then this will contradict Proposition 2.6 and complete the proof.

It remains to find a maximal ideal $M$ of $R$ such that $Q / Q M$ is not cyclic, and this seems to be considerably more subtle. We use the $L_{i}(Q)$, defined in $\S 1$, to find an appropriate ideal $M$. First note that, if $F$ is the field of fractions of $R$, then for all $i, L_{i}(Q) \otimes_{R} F$ is a cyclic ideal of $F[y]$. Since there are only finitely many distinct $L_{i}(Q)$ we can pick $c \in R \backslash\{0\}$ such that each $L_{i}(Q) \otimes_{R} R\left[c^{-1}\right]$ is cyclic. Since $L_{i}\left(Q\left[c^{-1}\right]\right)=L_{i}(Q) \otimes_{R} R\left[c^{-1}\right]$ we may, by replacing $R$ by $R\left[c^{-1}\right]$, assume that each $L_{i}(Q)$ is cyclic. Secondly let $I$ be the minimal ideal of $R$ such that $L_{\min }(Q) \subseteq I[y]$. Then, replacing $R$ by $R\left[d^{-1}\right]$ for any $d \in I \backslash\{0\}$, we may assume that $L_{i}(Q) \nsubseteq J[y]$ for any nonzero ideal $J$ of $R$ and nonzero $L_{i}(Q)$. (One consequence of this, which we do not use, is that for any maximal ideal $M$ of $R$ we may now identify $Q / Q M$ with $Q+M A / M A$.)

Since each $L_{i}(Q)$ is cyclic, with generator say $v_{i}$, we may pick $q_{i} \in Q$ such that $\operatorname{deg}\left(q_{i}\right)=i$ and $L_{i}\left(q_{i}\right)=v_{i}$. Since $Q$ is not cyclic, there exist $n>r$ such that $L_{n}(Q) \neq L_{r}(Q) \neq 0$. By enlarging $n$ if necessary, we may assume that $L_{n}(Q)=$ $L_{\infty}(Q)$. Thus for some maximal ideal $N$ of $R[y], L_{n}(Q) \neq L_{r}(Q)+L_{n}(Q) N$. Since $R$ is affine, $N$ contains a maximal ideal $M$ of $R$ and so $L_{n}(Q) \neq L_{r}(Q)+L_{n}(Q) M$. 
We will show that $Q / Q M$ is not cyclic, but we must first identify the $L_{i}(Q M)$. So, if $g \in Q M$ we may write $g=\sum g_{i} m_{i}$ for some $g_{i} \in Q$ and $m_{i} \in M$. But $g_{i}=$ $\sum q_{j} r_{i j}$ for some $r_{i j} \in R[y]$ and so $g=\sum_{0}^{s} q_{j}\left(\sum_{i} r_{i j} m_{i}\right)$, where after cancellation, we may assume that $\sum r_{i s} m_{i} \neq 0$. Thus $L(g) \in L_{s}(Q) M$. Hence for all $i$ we have $L_{i}(Q M)=L_{i}(Q) M$.

Now suppose that $Q=f A+Q M$, where $f \in Q$ is chosen to have minimal possible degree, say $\operatorname{deg}(f)=t$. Remember that $L_{t}(Q) \nsubseteq M[y]$. Thus if $L(f) \in M[y]$ then, by the last paragraph, $L(f)=v_{t} m$ for some $m \in M[y]$, and $Q=\left(f-q_{t} m\right) A+Q M$. Since $\operatorname{deg}\left(f-q_{t} m\right)<\operatorname{deg}(f)$, this contradicts the minimality of $\operatorname{deg}(f)$. Thus $L(f) \notin M[y]$. Next, write $q_{r}=f g+h$ for some $h \in Q M$ and $g \in A$, where we assume that $g$ has the smallest possible degree for this to happen. Note that if $L(g) \in M[y]$ then $f x^{\operatorname{deg}(g)} L(g) \in Q M$ and so $f g=f g_{1}+h_{1}$ for some $g_{1}$ with $\operatorname{deg} g_{1}<\operatorname{deg} g$; a contradiction. Thus $L(g) \notin M[y]$. But this implies that there can be no cancellation between the highest degree terms of $f g$ and $h$. Hence $\operatorname{deg} q_{r}=$ $\operatorname{deg}(f g+h) \geq \operatorname{deg} f+\operatorname{deg} g$. Thus $\operatorname{deg}(f) \leq r$ and $L(f) \in L_{r}(Q)$. Repeat the above argument for the equation $q_{n}=f a+b$. Again this ensures that there is no cancellation between the highest terms of $f a$ and $b$. Thus

$$
\begin{aligned}
L\left(q_{n}\right) & \in L(f) R[y]+L(b) R[y] \subset L_{r}(Q)+L_{\infty}(Q) M \\
& =L_{r}(Q)+L_{n}(Q) M .
\end{aligned}
$$

This contradicts the choice of $M$ and ensures that $Q / Q M$ is not cyclic. By the second paragraph of the proof of this step, this contradicts Proposition 2.6 and completes the proof of the theorern.

It is probably worth mentioning that, whatever the characteristic of $k, A=A(k)$ is stably free; that is, given any projective, finitely generated $A$-module $Q$ then $Q \oplus A^{(n)} \cong A^{(m)}$ for some integers $m$ and $n[\mathbf{7}$, Theorem 7]. It follows by [13] that $Q$ is free if it has uniform dimension $\geq 1+K \operatorname{dim} A$ (and $K \operatorname{dim} A=1$, respectively 2 if $\operatorname{char} k=0$, respectively $p>0$ ). In particular, given a projective right ideal $P$ of $A$ then $M_{n}($ End $P) \cong M_{n}(A)$ for any $n \geq 3$. If char $k=0$ this isomorphism also holds when $n=2$ and we would conjecture that the same is true for positive characteristic.

This means that there is a curious contrast between Theorem $\mathrm{A}$ and the commutative theory. For, if char $k=0$ then $A(k)$ is a Dedekind prime ring, which is the noncommutative analogue of a commutative Dedekind domain $C$. However, if $I$ is a noncyclic (right) ideal of $C$ then End $I=C$, yet certainly $I \oplus C^{(n)} \not C^{(m)}$ for any integers $n$ and $m$.

We next prove Corollary B of the introduction. If $d \in D(k) \backslash\{0\}$ then we will denote by $\sigma_{d}$ the inner automorphism determined by $d$; that is $\sigma_{d}(a)=d a d^{-1}$ for all $a \in D(k)$.

Corollary 3.2. Let $k$ be a field of any characteristic. Suppose that $P$ and $Q$ are projective right ideals of $A=A(k)$ such that $\operatorname{End} P \cong \operatorname{End} Q$. Then $Q=t \sigma(P)$ for some $t \in D(k)$ and $\sigma \in \operatorname{Aut}_{k}(A)$.

ProOF. We make the identifications of $\S 1$, so in particular End $P$ and End $Q$ are orders in $D(k)$. Thus, if $\theta$ : End $P \rightarrow$ End $Q=S$ is the given isomorphism, then $\theta$ may be considered as an automorphism of $D(k)$. Note that $\theta(P)$ is now a left $S$-module. 
We consider $T=Q^{*} \theta(P) \cong Q^{*} \otimes_{S} \theta(P)$. This is certainly a projective left $A$-module and a right $\theta(A)$-module. By (1.1), $\theta(A)=\operatorname{End}\left({ }_{A} T\right)$. Thus, applying Theorem 3.1 to the left $A$-module $T$, we have $T=A t$ for some $t \in D(k)$. Thus

$$
t^{-1} A t=\operatorname{End}(A t)=\operatorname{End}(T)=\theta(A) .
$$

In particular, $A=\sigma_{t} \theta(A)$ and $\sigma=\sigma_{t} \theta \in$ Aut $A$. Finally, as sets,

$$
Q t=Q T=Q Q^{*} \theta(P)=S \theta(P)=\theta(P)=t^{-1} \sigma(P) t .
$$

Thus $Q=t^{-1} \sigma(P)$; as required.

A curious consequence of Corollary 3.2 is that $\operatorname{Aut}_{k}(\operatorname{End} P)$ is "induced" from $\operatorname{Aut}_{k}(A)$. Another way of obtaining $\operatorname{Aut}_{k}\left(\right.$ End $P$ ) from $\operatorname{Aut}_{k}(A)$ will be given in Theorem 4.7.

COROLlaRY 3.3. Let $S$ be a domain that is Morita equivalent to $A=A(k)$. Then for all $\theta \in \operatorname{Aut}_{k}(S)$ there exists $d \in D(k)$ and $\sigma \in \operatorname{Aut}_{k}(A)$ such that $\theta=\sigma_{d} \sigma$.

Proof. By hypothesis, we may identify $S$ with $\operatorname{End}(Q)$ for some projective right ideal $Q$ of $A$. If we set $P=Q$, then $\theta \in \operatorname{Aut}_{k}(S)$ gives an isomorphism between End $P$ and End $Q$. Thus, by the proof of Corollary 3.2, $\theta=\sigma_{d} \sigma$ for some $d \in D(k)$ and $\sigma \in \operatorname{Aut}_{k}(A)$.

As will be shown in the next section, if $S$ is not isomorphic to $A$, then the converse to Corollary 3.3 is false. That is, there exists $\phi \in \operatorname{Aut}_{k}(A)$ such that $\phi \neq \sigma_{d} \tau$ for any $d \in D(k)$ and $\tau \in \operatorname{Aut}_{k}(S)$.

4. Rings Morita equivalent to the Weyl algebra. Throughout this section $k$ will denote a field of arbitrary characteristic and $A=A(k)$. The purpose of this section is to show that the results of the last two sections are specific to the Weyl algebra, in the sense that they all become false if $A$ is replaced by any domain $R$ that is Morita equivalent but not isomorphic to $A$. Thus, while $A$ and $R$ have equivalent module categories, they have surprisingly different properties. This can be best expressed by using a canonical coset space, $\overline{\operatorname{Pic}_{k}}(S)=\operatorname{Pic}_{k}(S) / \operatorname{Im}\left(\operatorname{Aut}_{k}(S)\right)$ of the Picard group of a ring $S$ (the definition will be given later). For, Theorem A just says that $\overline{\operatorname{Pic}_{k}}(A)=\{1\}$. In contrast, at least when $k$ is infinite, we prove that $\overline{\operatorname{Pic}_{k}}(R)$ is infinite. This will also mean that other positive results for $A$, like Theorem 2.4 and Corollary 3.3, will fail for $R$.

The reason why this happens is really that Corollary $\mathrm{B}$, while containing Theorem $\mathrm{A}$ as a special case, is a weaker result, in that one needs to involve $\operatorname{Aut}_{k}(A)$. The following easy example shows that one cannot strengthen Corollary B. Let $P_{1}=x^{2} A+(x y+1) A$ be the 'canonical' noncyclic projective $A$-module and $Q=x^{2} A+(x(y+1)+1) A$. Then certainly $Q=\sigma\left(P_{1}\right)$ for the appropriate $\sigma \in \operatorname{Aut}_{k}(A)$ but it is readily proven that $Q \neq t P_{1}$ for any $t \in D(k)$. Thus one cannot strengthen Corollary B, and this easily implies that Theorem A fails if we replace $A$ by $\operatorname{End}\left(P_{1}\right)$. We remark that $P_{1}$ provides a useful test case for the results of this section, as the modus operandi will be to mimic, for an arbitrary projective module, proofs that are easy for $P_{1}$.

We begin with two preparatory lemmas. The first justifies the earlier comment that the statement of Corollary B is weaker than that of Theorem A and the second shows that one crucial property of $P_{1}$, that it intersects $k[x]$, can be assumed for an arbitrary projective module. 
LEMma 4.1. Let $Q$ be a noncyclic, projective right ideal of $A$ and put $S=$ $\operatorname{End}(Q)$. Suppose that there exists $\sigma \in \operatorname{Aut}_{k}(A)$ such that $\sigma(Q) \neq t Q$ for any $t \in D(k)$. Then there exists a noncyclic, projective right ideal $M$ of $S$ such that $S \cong \operatorname{End}(M)$. Here $M=s \sigma(Q) Q^{*}$ for some $s \in S$.

Proof. As usual, we identify $\operatorname{End}(Q)$ with a subalgebra of $D(k)$. Since $\sigma \in$ Aut $(A), \sigma(Q)$ is a right ideal of $A$ and regarding $\sigma$ as an element of Aut $(D(k))$, we have $\sigma(S)=\operatorname{End}\left(\sigma(Q)_{A}\right)$. Put

$$
M=\sigma(Q) Q^{*} \cong \sigma(Q) \otimes_{A} Q^{*} .
$$

Then $M$ is a projective right $S$-submodule of $D(k)$, and so is isomorphic to a right ideal of $S$. Further, using (1.1), $\operatorname{End}\left(M_{S}\right)=\sigma(S) \cong S$. Suppose that $M=t S$ for some $t \in D(k)$. Then

$$
\sigma(Q)=\sigma(Q) A=\sigma(Q) Q^{*} Q=t S Q=t Q ;
$$

contradicting our hypothesis. Thus $M$ is not cyclic, as required.

Lemma 4.2. Let $Q$ be a nonzero right ideal of $A$. Then there exists $t \in$ $D(k) \backslash\{0\}$ such that $t Q$ is a right ideal of $A$ that satisfies $t Q \cap k[x] \neq 0$.

Proof. Let $C=k[x] \backslash\{0\}$. Then, as is well known and easy to check, $C$ is an Ore set in $A$ and $B=A_{\mathcal{C}}=k(x)\langle y\rangle$ is a principal ideal domain. Thus $Q_{C}=q A_{\mathcal{C}}$ for some $q \in Q$. Now $q^{-1} Q$ is a finitely generated right $A$-module satisfying $A \subseteq q^{-1} Q \subseteq A_{C}$. Thus we may clear denominators (on the left) and obtain $g A \subseteq g q^{-1} Q \subseteq A$ for some $g \in C$. Thus $t=g q^{-1}$ satisfies the conclusions of the lemma.

THEOREM 4.3. Let $k$ be an infinite field and $Q$ a noncyclic, projective right ideal of $A=A(k)$. Put $S=\operatorname{End} Q$. Then there exists a noncyclic, projective right ideal $M$ of $S$ such that $S \cong \operatorname{End}\left(M_{S}\right)$.

REMARK 4.4. We actually prove more. Given $\lambda \in k$, define $\theta_{\lambda} \in \operatorname{Aut}_{k}(A)$ by $\theta_{\lambda}(x)=x$ and $\theta_{\lambda}(y)=y+\lambda$. Set $M\left(\theta_{\lambda}\right)=\theta_{\lambda}(Q) Q^{*}$. Then we prove, for all but finitely many $\lambda \in k$, that $M\left(\theta_{\lambda}\right)$ is a noncyclic projective $S$-module for which $S \cong \operatorname{End}\left(M\left(\theta_{\lambda}\right)\right)$.

ProOF. By Lemma 4.2 pick $t \in D(k)$ such that $t Q$ is a right ideal of $A$ for which $t Q \cap k[x] \neq 0$. Since $\operatorname{End}(t Q)=t S t^{-1} \cong S$ it is sufficient to prove the theorem for $t Q$. Note that, in the notation of Remark 4.4, if $\theta_{\lambda}(Q) Q^{*}=v S$ is a cyclic $S$-module, then

$$
\theta_{\lambda}(t Q)(t Q)^{*}=\theta_{\lambda}(t) \theta_{\lambda}(Q) Q^{*} t^{-1}=\left[\theta_{\lambda}(t) v t^{-1}\right]\left(t S t^{-1}\right)
$$

is a cyclic $t S t^{-1}$-module. Thus the remark is also unaffected by this change.

Thus we may assume that $Q \cap k[x] \neq 0$. In order to prove the theorem and the remark, Lemma 4.1 says that it suffices to prove that, for all but finitely many $\lambda \in k$, we have $\theta_{\lambda}(Q) \neq s Q$ for any $s \in D(k)$. In the notation of $\S 1$, consider the $L_{i}(Q)$. As $Q \cap k[x] \neq 0$, we have $L_{\infty}(Q)=k[y]=L_{n}(Q)$ for some $n$. Pick $r$ minimal such that $L_{r}(Q) \neq 0$; say $L_{r}(Q)=f k[y]$. Note that, by (1.5), $f \notin k$. Thus, for all but finitely many $\lambda \in k$, we have $\theta_{\lambda}(f) \neq f$, and, therefore, $\theta_{\lambda}(f) \neq v f$ for any $v \in k$. For any $a \in A$, $\operatorname{deg} \theta_{\lambda}(a)=\operatorname{deg} a$ and $L\left(\theta_{\lambda}(a)\right)=\theta_{\lambda}(L(a))$. Thus $L_{i}\left(\theta_{\lambda}(Q)\right)=k[y]$ for $i \geq n$, while

$$
L_{r}\left(\theta_{\lambda}(Q)\right)=\theta_{\lambda}(f) k[y] \neq L_{r}(Q) .
$$


Now, suppose that $\theta_{\lambda}(Q)=s Q$ for some $s \in D(k)$. Equivalently, $a \theta_{\lambda}(Q)=b Q$ for some $a, b \in A \backslash\{0\}$. For $i \gg 0$,

$$
L_{i}\left(a \theta_{\lambda}(Q)\right)=L(a) L_{i-\operatorname{deg}(a)}\left(\theta_{\lambda}(Q)\right)=L(a) k[y]
$$

while $L_{i}(b Q)=L(b) k[y]$. Thus $L(a)=\mu L(b)$ for some $\mu \in k \backslash\{0\}$. On the other hand, by comparing nonzero elements of minimal degree in the equation $a \theta_{\lambda}(Q)=b Q$ we see that $\operatorname{deg} a=\operatorname{deg} b$ and

$$
L(a) \theta_{\lambda}(f) k[y]=L_{r+\operatorname{deg} a}\left(a \theta_{\lambda}(Q)\right)=L(b) f k[y]=L(a) f k[y] .
$$

Thus $\theta_{\lambda}(f)=v f$ for some $v \in k\{0\}$. This contradicts the choice of $\lambda$ and completes the proof of the theorem.

REMARK. It is not clear whether the theorem remains true over a finite field, as there seems to be no reasonable infinite set of automorphisms to use in place of the $\theta_{\lambda}$. The trouble with the more general automorphisms $\sigma_{i f}$ of Corollary 2.8 is that the $L_{j}\left(\sigma_{2 g}(Q)\right)$ become almost impossible to compute when $g \notin k$, while $L_{j}\left(\sigma_{1 f}(Q)\right)=L_{j}(Q)$ for any $f$.

The dichotomy between Theorems 3.1 and 4.3 suggest that there should be an obvious invariant that distinguishes between $A(k)$ and $S=\operatorname{End}(Q)$. The most natural such invariant arises from the Picard group. For a $k$-algebra $R$, the Picard group of $R$ relative to $k$, written $\mathrm{Pic}_{k} R$, is the group of all bimodule isomorphism classes of invertible $R$-bimodules over $k$, with multiplication defined by tensor product. Here, an $\left(R_{1}, R\right)$-bimodule $M$ is invertible if $M \otimes_{R}$-defines an equivalence of categories between the categories of $R$-modules and $R_{1}$-modules. That it is a bimodule over $k$ means that $g m=m g$ for all $m \in M$ and $g \in k$. For the rings $R$ that concern us, $R$ is a domain and every finitely generated projective $R$-module is stably free and hence a progenerator. Thus an invertible $R$-bimodule $M$ over $k$ is just a projective right ideal of $R$ such that $R \cong \operatorname{End}(M)$ as $k$-algebras. The details concerning Picard groups can be found, for example, in [8, §37]. The Picard group itself is not appropriate for our purposes since it is a Morita invariant (see [8, Theorem 37.9]). However, by [8, Theorem 37.14] there is a natural homomorphism $\Omega: \operatorname{Aut}_{k}(R) \rightarrow \operatorname{Pic}_{k}(R)$ defined as follows. Given an $R$-bimodule, $M$ and automorphisms $\sigma, \tau \in \operatorname{Aut}_{k}(R)$ define ${ }_{\sigma} M_{\tau}$ to be the $R$-bimodule, isomorphic to $M$ as an abelian group but with multiplication twisted by the automorphisms; $r \circ m \circ s=\sigma(r) m \tau(s)$ for $r, s \in R$ and $m \in M$. Then $\Omega$ is defined by $\left.\Omega(\sigma)={ }_{1} R_{\sigma}\right]=\left[{ }_{\bar{\sigma}} R_{1}\right]$. Here and elsewhere we write $\bar{\sigma}$ for $\sigma^{-1}$ to avoid cumbersome notation. Theorems 3.1 and 4.3 can now be translated into results about Picard groups to give

COROLlary 4.5. (i) If $R \cong A$, then $\operatorname{Pic}_{k}(R)=\operatorname{Im}(\Omega)$.

(ii) If $k$ is an infinite field and $R$ is a domain that is Morita equivalent but not isomorphic to $A$, then $\operatorname{Pic}_{k}(R) \neq \operatorname{Im}(\Omega)$.

PROOF. (i) By [8, Theorem 37.16], $\operatorname{Im}(\Omega)$ consists of those invertible $R$-bimodules that are cyclic as right modules. But Theorem 3.1 says that invertible $A$ bimodules over $k$ are also cyclic as right modules; as required.

(ii) By Theorem 4.3, there exists an invertible $R$-bimodule that is not cyclic as a right $R$-module, and so again the result follows from [8, Theorem 37.16].

REMARK. Another proof of Corollary 3.2 can be given by combining Corollary 4.5(i) with [1, Corollary 3.3]. Also, by combining Corollary 4.5(i) with [1, 
Proposition 1.6] one obtains that for $n \geq 2, \operatorname{Pic}_{k}\left(M_{n}(A)\right)=\operatorname{Im}(\Omega)$. Note that, by the comments after Theorem 3.1, every essential projective right ideal of $M_{n}(A)$ is free if $n \geq 3$ (and this also holds for $n=2$ if char $k=0$ ). Thus the fact that Pic $M_{n}(A)=\operatorname{Im}(\Omega)$ is immediate in this case. Of course, as $M_{n}(A) \cong M_{n}(R)$ for $n \geq 3$, Corollary 4.5(ii) shows that the converse of [1, Proposition 1.6] does not hold.

Our next aim is to refine Corollary 4.5 by showing that $\Omega$ is always a monomorphism and that, in the situation of Corollary $4.5(\mathrm{ii}), \operatorname{Im}(\Omega)$ has infinite index in $\operatorname{Pic}_{k}(R)$.

LEMMA 4.6. Let $Q$ be a projective right ideal of $A$ and put $S=\operatorname{End}(Q)$. Then

(i) if $U(S)$ denotes the units of $S$, then $U(S)=k \backslash\{0\}$;

(ii) the homomorphism $\Omega: \operatorname{Aut}_{k}(S) \rightarrow \operatorname{Pic}_{k}(S)$ is a monomorphism.

REMARK. It is a triviality that $U(A)=k \backslash\{0\}$. However one cannot prove part (i) of the lemma just by using this and the fact that $A$ and $S$ are Morita equivalent domains, as can be seen by considering the result of Paramila [6] that was mentioned in the introduction.

PROOF. By [8, Theorem 37.14], $\operatorname{Ker}(\Omega)=\operatorname{Inn}_{k}(S)$, the set of inner automorphisms of $S$. Thus (ii) follows from (i).

By Lemma 4.2, there exists $t \in D(k)$ such that $t Q$ is a right ideal of $A$ for which $t Q \cap k[x] \neq 0$. Note that $\operatorname{End}(t Q) \cong \operatorname{End}(Q)$ and so $U(\operatorname{End} Q)=k \backslash\{0\}$ if and only if $U(\operatorname{End}(t Q))=k \backslash\{0\}$. Thus we may replace $Q$ by $t Q$. Set $Q \cap k[x]=h k[x]$. If $B=k(x)\langle y\rangle$, in the notation of Lemma 4.2, then End $Q \subset Q^{*} \subseteq A h^{-1} \subset B$. Thus $U($ End $Q) \subseteq U(B)=k(x) \backslash\{0\}$. If $s \in U($ End $Q)$ we may therefore write $s=f^{-1} g$ for some $f, g \in k[x]$. But $s Q=Q$ implies that $f Q=g Q$ and hence that

$$
f h k[x]=f(Q \cap k[x])=f Q \cap k[x]=g Q \cap k[x]=g h k[x] .
$$

Thus $s=f^{-1} g \in k \backslash\{0\}$; as required.

We now turn to the coset space $\operatorname{Pic}(S) / \operatorname{Im}(\Omega)$, where $S=\operatorname{End}(Q)$ is the endomorphism ring of a noncyclic, projective right ideal $Q$ of $A$. It will follow from Remark 4.4 that this coset space is infinite, but to show this we need a different description of the $M\left(\theta_{\lambda}\right)$ of Remark 4.4. Remember that, if $\theta \in \operatorname{Aut}_{k}(A)$ then $M(\theta)=\theta(Q) Q^{*} \cong \theta(Q) \otimes_{A} Q^{*}$. Of course $\operatorname{End}\left(M(\theta)_{S}\right)=\theta(S)$ and so $M(\theta)$ becomes an invertible $S$-bimodule under the action $s \circ m \circ t=\theta(s) m t$ for $s, t \in S$ and $m \in M(\theta)$.

It is now routine to show that there is an $S$-bimodule isomorphism $\alpha: M(\theta) \rightarrow$ $\left({ }_{1} Q_{\bar{\theta}}\right) \otimes_{A} Q^{*}$ given by $\alpha(\theta(q) \otimes f)=q \otimes f$ for $q \in Q$ and $f \in Q^{*}$. Furthermore, ${ }_{1} Q_{\bar{\theta}} \cong Q \otimes_{A}\left({ }_{1} A_{\bar{\theta}}\right)$ as $(S, A)$-bimodules, and so $M(\theta) \cong Q \otimes_{A}\left({ }_{1} A_{\bar{\theta}}\right) \otimes_{A} Q^{*}$ as $S$-bimodules. The reason for writing $M(\theta)$ in this way, is that combining Corollary 4.5(i) and Lemma 4.6 with [8, Theorems 37.14 and 37.9] we now have isomorphisms $\operatorname{Aut}_{k}(A) \rightarrow \operatorname{Pic}_{k}(A) \rightarrow \operatorname{Pic}_{k}(S)$ given by

$$
\theta \rightarrow\left[{ }_{1} A_{\theta}\right] \rightarrow\left[Q \otimes_{A}\left({ }_{1} A_{\theta}\right) \otimes_{A} Q^{*}\right]=[M(\bar{\theta})] .
$$

In particular, using the notation of Remark 4.4, there is a group monomorphism $\beta$ from the additive group of $k$ to $\operatorname{Pic}_{k}(S)$, given by $\beta(\lambda)=\left[M\left(\theta_{-\lambda}\right)\right]$. Finally, Remark 4.4 says that $\operatorname{Im}(\beta) \cap \operatorname{Im}(\Omega)$ is a finite group. 
Combining these observations with the earlier results on the Picard group we have

THEOREM 4.7. Let $k$ be an infinite field and $Q$ a noncyclic projective right ideal of $A=A(k)$. Set $S=\operatorname{End}(Q)$. Then $\operatorname{Aut}_{k}(A) \cong \operatorname{Pic}_{k}(A)$ whereas $\Omega$ : $\operatorname{Aut}_{k}(S) \rightarrow$ $\operatorname{Pic}_{k}(S) \cong \operatorname{Pic}_{k}(A)$ is a monomorphism such that the coset space $\operatorname{Pic}_{k}(S) / \operatorname{Im}(\Omega)$ has cardinality greater than or equal to card $(k)$.

This theorem raises the obvious questions of whether one can reasonably describe $\operatorname{Aut}_{k}(S)$ and whether there exists some canonical complementary subgroup to $\operatorname{Aut}_{k}(S)$ in $\mathrm{Pic}_{k}(S)$. (We suspect that $\operatorname{Aut}_{k}(S)$ will never be a normal subgroup of $\operatorname{Pic}_{k}(S)$.) Note that, by [3, Théorème 8.10] and [5, Theorem 1], $\operatorname{Aut}_{k}(A(k))$ has been calculated for all fields $k$, and it would be interesting to know whether there exists a similar description of $\operatorname{Aut}_{k}(S)$. Indeed, we do not even know whether $\operatorname{Aut}_{k}(S) \not \operatorname{Aut}_{k}(A)$ as abstract groups.

We end the section with two further results that illustrate the difference between $\operatorname{Aut}_{k}(A)$ and $\operatorname{Aut}_{k}(S)$. The first should be compared with Theorem 2.4 and the second with Corollary 3.3.

COROLlaRY 4.8. Let $k$ be an infinite field with $\operatorname{char}(k)>0$ and $S$ a domain that is Morita equivalent, but not isomorphic to $A$. Then there exists an automorphism $\sigma$ of the center $Z(S)$ that lifts to an automorphism of $D(k)$ but does not lift to an automorphism of $S$.

ProOF. We remark that $D(k)$ is also the quotient division ring of $S$. By Theorem 4.3, pick a noncyclic, projective right ideal $M$ of $S$ such that there exists an isomorphism $\tau: S \rightarrow \operatorname{End}\left(M_{S}\right)$. Then certainly $\tau$ restricts to an automorphism $\sigma$ of $Z(S)=Z($ End $M)$ and extends to an automorphism of $D(k)$. But if $\sigma$ extends to an automorphism of $S$, then the proof of Proposition 2.6 may be used to imply that $M$ is cyclic; a contradiction.

COROLlary 4.9. Let $k$ be an infinite field and $S$ a domain that is Morita equivalent but not isomorphic to $A$. Then there exists $\sigma \in \operatorname{Aut}_{k}(A)$ such that $\sigma \neq \sigma_{d} \tau$ for any $d \in D(k)$ and $\tau \in \operatorname{Aut}_{k}(S)$.

Proof. Write $S=\operatorname{End}(Q)$ for an appropriate projective right ideal $Q$ of $A$. By Remark 4.4 there exists $\sigma \in \operatorname{Aut}_{k}(A)$ such that $M=\sigma(Q) Q^{*}$ is a noncyclic, projective right $S$-module. Suppose that $\sigma=\sigma_{d} \tau$ for some $d \in D(k)$ and $\tau \in$ $\operatorname{Aut}_{k}(S)$. Then

$$
N=d^{-1} M=d^{-1}\left(d \tau(Q) d^{-1}\right) Q^{*}=\tau(Q) d^{-1} Q^{*}
$$

is also a noncyclic right $S$-module. However, by $(1.1), \operatorname{End}\left(N_{S}\right)=\tau(S)=S$. Thus $N$ is an $S$-bisubmodule of $D(k)$ that is finitely generated and projective on both sides. This forces $N_{S}$ to be cyclic; if $\operatorname{char}(k)>0$ use the last part of the proof of Proposition 2.6 while if $\operatorname{char}(k)=0$ use the fact that $S$ is simple and hence that the only $S$-bisubmodule of $D(k)$ that is finitely generated as a one-sided module is $S$ itself.

We remark that (under the appropriate conditions on $k$ ) Corollary 4.9 also follows from Corollary 4.8 combined with Theorem 2.4. The proof is left to the interested reader. 
5. Nonisomorphism of endomorphism rings. An obvious question raised by Theorem 3.1 is whether there exist infinitely many domains that are Morita equivalent to $A=A(k)$, but are pairwise nonisomorphic. For example, this ought to be the case for the $\operatorname{End}\left(P_{n}\right)$ where $P_{n}=x^{n+1} A+(x y+n) A$. Unfortunately we can say almost noting about this problem, although we do at least show that it is a reasonable conjecture by confirming that $\operatorname{End}\left(P_{1}\right) \not \equiv \operatorname{End}\left(P_{i}\right)$ for $i>1$.

Actually, we phrase the result rather differently by stating it in terms of rings of differential operators. Throughout this section $X$ and $Y$ will denote irreducible, affine algebraic curves over an algebraically closed field $k$ of characteristic zero. Then the ring of differential operators $D(X)$ on $X$ is defined as follows. Set $D^{0}(X)=$ $\mathcal{O}(X)$, the ring of regular functions on $X$ and, for $n>0$,

$$
D^{n}(X)=\left\{\theta \in \operatorname{Hom}_{k}(O(X), O(X)):[\theta, a] \in D^{n-1} \text { for all } a \in O(X)\right\} .
$$

Then $D(X)=\bigcup D^{n}(X)$. All the results that we need about $D(X)$ can be found in [12]. A natural question is whether $D(X)$ determines $X$ in the sense that $D(X) \cong$ $D(Y)$ implies that $X \cong Y$. This is closely related to the question with which we began this section. For, suppose from now on that $X$ has normalization $\tilde{X} \cong \mathrm{A}$, the affine line over $k$. Then $D(\tilde{X}) \cong A=A(k)$. Under this identification $\mathcal{O}(\tilde{X})=$ $k[x] \subset A$ and $\partial / \partial x=-y$. In order to avoid confusion with multiplication in $A$, the action of a differential operator $\theta \in D(X)$ on an element $a \in O(X)$ will be denoted by $\theta * a$. Now define

$$
P=D(\tilde{X}, X)=\{\theta \in D(\tilde{X}): \theta * O(\tilde{X}) \subseteq O(X)\} .
$$

Then $P$ is a right ideal of $A$. Furthermore, $D(X) \subseteq \operatorname{End}\left(P_{A}\right)$ with equality if and only if $D(X)$ is simple [12, Proposition 3.3]. Translating Theorem 3.1 into this context gives

Proposition 5.1. Let $X$ be as above, with $\tilde{X}=A$. Then $D(X) \cong D(\tilde{X})$ if and only if $X=\tilde{X}$.

Proof. Suppose that $X \neq \tilde{X}$. If $D(X) \cong D(\tilde{X})=A$, then $D(X)$ is simple and so, by the above comments, $D(X)=\operatorname{End}(P)$. Thus, by Theorem 3.1, $D(\tilde{X}, X)=f A$ for some $f \in A$. Since $O(X) \supset c \mathcal{O}(\tilde{X})$ for some nonzero $c \in \mathcal{O}(\tilde{X})$, certainly $c A \subset P$. As $c \in k[x]$ this forces $f \in k[x]$. But now

$$
D(\tilde{X}, X) * k[x]=f A * k[x]=f k[x] \neq \mathcal{O}(X) .
$$

This contradicts the equivalence of parts (b) and (c) of [12, Proposition 3.3] and completes the proof.

We now turn to the problem of whether $D(X) \cong D(Y)$ implies that $X \cong Y$. As slight evidence in favour of the conjecture we show that this is the case when $X$ is the cubic cusp, $Z_{1}^{2}=Z_{2}^{3}$. In this case $O(X)=k\left[x^{2}, x^{3}\right]$ and $P=D(\tilde{X}, X)=x^{2} A+$ $(x y+1) A$, by $[\mathbf{1 2}, \S 3.8]$. Further, $[\mathbf{1 2}$, Theorem 3.4] implies that $D(X)=\operatorname{End}(P)$. Note that $P=P_{1}$ in the notation of the first paragraph of this section.

We intend to apply Corollary 3.2 to prove that $D(X) \not \equiv D(Y)$ whenever $Y \not X X$, but to do so we must first understand $\sigma(P)$ when $\sigma \in \operatorname{Aut}_{k}(A)$. For $\lambda, \mu \in k$ define

$$
P_{\lambda, \mu}=(x+\lambda)^{2} A+((x+\lambda)(y+\mu)+1) A .
$$


An elementary, but useful calculation shows that

$$
P_{\lambda, \mu}=A \cap(y+\mu)^{-1}(x+\lambda) A .
$$

LEMma 5.2. Let $\sigma \in \operatorname{Aut}_{k}(A)$ and $\lambda, \mu \in k$. Then $\sigma\left(P_{\lambda, \mu}\right)=t P_{\alpha, \beta}$ for some $\alpha, \beta \in k$ and $t \in D(k)$.

ProOF. By [3, Théorème 8.10], $\operatorname{Aut}_{k}(A)$ is generated by the $\sigma_{i f}$ of Corollary 2.8 and so it suffices to prove the lemma for these automorphisms. Suppose first that $\sigma=\sigma_{2 g}$; so $g \in k[x]$ and we may write $g=\alpha+(x+\lambda) g_{1}$ for some $\alpha \in k$ and $g_{1} \in k[x]$. Then

$$
\begin{aligned}
\sigma_{2 g}\left(P_{\lambda, \mu}\right) & =(x+\lambda)^{2} A+\left\{(x+\lambda)\left(y+\mu+\alpha+(x+\lambda) g_{1}\right)+1\right\} A \\
& =(x+\lambda)^{2} A+\{(x+\lambda)(y+\mu+\alpha)+1\} A=P_{\lambda, \mu+\alpha} .
\end{aligned}
$$

Now suppose that $\sigma=\sigma_{1 f}$ for some $f \in k[y]$ and write $f=\alpha+(y+\mu) f_{1}$ for some $\alpha \in k$ and $f_{1} \in k[y]$. In this case

$$
\begin{aligned}
P_{\lambda, \mu} & =A \cap(y+\mu)^{-1}(x+\lambda) A \cong(x+\lambda)^{-1}(y+\mu) A \cap A \\
& =(y+\mu)^{2} A+((y+\mu)(x+\lambda)-1) A=Q_{\mu, \lambda} .
\end{aligned}
$$

Thus $\sigma_{1 f}\left(P_{\lambda, \mu}\right) \cong \sigma_{1 f}\left(Q_{\mu, \lambda}\right)=Q_{\mu, \lambda+\alpha} \cong P_{\lambda+\alpha, \mu}$. Therefore $\sigma_{1 f}\left(P_{\lambda, \mu}\right)=t P_{\lambda+\alpha, \mu}$ for some $t \in D(k)$; as required.

PROPOSITION 5.3. Let $X$ and $Y$ be two nonisomorphic curves such that $\tilde{X} \cong$ $\tilde{Y} \cong \mathrm{A}$. Then, provided that $X$ is the cubic cusp $Z_{1}^{2}=Z_{2}^{3}$, we have $D(X) \neq D(Y)$.

Proof. Suppose that $D(X) \cong D(Y)$. As remarked earlier, $D(X)=\operatorname{End}_{A}(P)$ is a simple ring and so, by [12, Proposition 3.3] again, $D(Y)=\operatorname{End}_{A}(Q)$ for $Q=$ $D(\tilde{Y}, Y)$. By Corollary 3.2, $Q=s \sigma(P)$ for some $s \in D(K)$ and $\sigma \in \mathrm{Aut}_{k}(A)$. By Lemma 5.2, $Q=t P_{\lambda, \mu}$ for some $\lambda, \mu \in k$ and $t \in D(k)$. Now, the set $C=\left\{(x+\lambda)^{n}\right\}$ is an Ore set in $A$ and $\left(P_{\lambda, \mu}\right)_{\mathcal{C}}=A_{\mathcal{C}}$. Thus $Q_{\mathcal{C}}=t A_{\mathcal{C}}$. As $Q_{\mathcal{C}} \subseteq A_{\mathcal{C}}$ this forces $t \in A_{\mathcal{C}}$; say $t=(x+\lambda)^{-n} u$ for some $u \in A$ and $n \in \mathbb{N}$. We may clearly suppose that $n \geq 2$. Since $Q=D(\tilde{Y}, Y)$, clearly $Q \cap k[x]=\operatorname{ann}_{\mathcal{O}(Y)}(\mathcal{O}(\tilde{Y}) / \mathcal{O}(Y))$, the conductor of $O(Y)$ in $O(\tilde{Y})$. In particular $Q \cap k[x] \neq 0$; say $Q \cap k[x]=c k[x]$.

From the equation $(x+\lambda)^{n} Q=u P_{\lambda, \mu}$ we obtain

$$
u P_{\lambda, \mu} \cap k[x]=(x+\lambda)^{n} Q \cap k[x]=(x+\lambda)^{n} c k[x] .
$$

This forces $u \in k[x]$ and $u P_{\lambda, \mu} \cap k[x]=u\left(P_{\lambda, \mu} \cap k[x]\right)=u(x+\lambda)^{2} k[x]$. Thus $u(x+\lambda)^{2}=(x+\lambda)^{n} c v$ for some $v \neq 0 \in k$. Thus $u=(x+\lambda)^{n-2} c v$ and $(x+\lambda)^{2} Q=$ $c P_{\lambda, \mu}$. Now compute the action of the two sides of this equation on $O(\tilde{X})=k[x]$. Since $D(Y)$ is simple, [12, Proposition 3.3] implies that $Q * k[x]=\mathcal{O}(Y)$. Thus

$$
(x+\lambda)^{2} \in(x+\lambda)^{2} O(Y)=(x+\lambda)^{2} Q * k[x]=c P_{\lambda, \mu} * k[x] \subseteq c k[x] .
$$

But, by construction, $c k[x] \subseteq \mathcal{O}(Y)$ and so $(x+\lambda)^{2} k[x] \subseteq \mathcal{O}(Y)$. Thus, either $O(Y)=O(\tilde{Y})$--which contradicts Proposition 5.1, or $O(Y)=(x+\lambda)^{2} k[x]+k$-in which case $O(Y) \cong O(X)$; as required.

We began this section by considering $\operatorname{End}\left(P_{n}\right)$ for $P_{n}=x^{n+1} A+(x y+n) A$. It is easy to modify the proof of Proposition 5.3 to prove that $\operatorname{End}\left(P_{1}\right) \not \nexists \operatorname{End}\left(P_{i}\right)$ for $i \geq 1$. In fact this also follows directly since we can express the $\operatorname{End}\left(P_{i}\right)$ as rings of differential operators. For, let $X_{n}$ be the curve for which $\mathcal{O}\left(X_{n}\right)=k+x^{n+1} k[x]$. 
Then $\left[\mathbf{1 2}\right.$, Theorem 3.4] implies that $D\left(X_{n}\right)=\operatorname{End}\left(Q_{n}\right)$ where $Q_{n}=D\left(\tilde{X}_{n}, X_{n}\right) \subset$ $A$. However if $\sigma \in \operatorname{Aut}_{k}(A)$ is defined by $\sigma(x)=y$ and $\sigma(y)=-x$, then one can prove that

$$
D\left(\tilde{X}_{n}, X_{n}\right)=A \cap y^{-1} x^{n} A=y^{-1} x^{n} \sigma\left(A \cap y^{-n} x A\right)=y^{-1} x^{n} \sigma\left(P_{n}\right) .
$$

Thus $D\left(X_{n}\right) \cong \operatorname{End}\left(P_{n}\right)$.

Finally, the computations of this section can be used to improve the results of the last section for the special case of $S_{1}=\operatorname{End}\left(P_{1}\right)$. For, it follows easily from Lemma 5.2 that a transversal for $\operatorname{Aut}_{k}\left(S_{1}\right)$ in $\operatorname{Pic}_{k}\left(S_{1}\right)$ is given by the $M\left(\sigma_{\lambda, \mu}\right)=$ $\sigma_{\lambda, \mu}\left(P_{1}\right) P_{1}^{*}$. Here $\lambda, \mu \in k$ and $\sigma_{\lambda, \mu}$ is defined by $\sigma_{\lambda, \mu}(x)=(x+\lambda)$ and $\sigma_{\lambda, \mu}(y)=$ $(y+\mu)$. Also one can use this to prove that $\operatorname{Aut}_{k}\left(S_{1}\right)$ is not a normal subgroup of $\operatorname{Pic}_{k}\left(S_{1}\right)$.

\section{REFERENCES}

1. M. L. Bolla, Isomorphisms between endomorphism rings of progenerators, J. Algebra 87 (1984), 261-281.

2. M. Chamarie, Modules sur les anneaux de Krull non commutatifs, Séminaire d'algèbre P. Dubreil et M.-P. Malliavin, Lecture Notes in Math., no. 1029, Springer-Verlag, Berlin and New York, 1983.

3. J. Dixmier, Sur les algèbres de Weyl, Bull. Soc. Math. France 96 (1968), 209-242.

4. K. R. Goodearl, Centralisers in differential, pseudodifferential and fractional differential operator rings, Rocky Mountain J. Math. 13 (1983), 573-618.

5. L. Makar-Limanov, On automorphisms of Weyl algebra, Bull. Soc. Math. France 112 (1985), 359-363.

6. R. Paramila, Automorphisms of non-free projective ideals of polynomial extensions of division rings, Arch. Math. (Basel) 38 (1982), 531-534.

7. D. Quillen, Higher algebraic K-theory, Algebraic K-theory. I: Higher K-theories, Lecture Notes in Math., no. 341, Springer-Verlag, Berlin and New York, 1973.

8. I. Reiner, Maximal orders, Academic Press, London and New York, 1975.

9. P. Revoy, Algèbres de Weyl en charactéristique p, C. R. Acad. Sci. Paris 276 (1973), A225-A228.

10. J. J. Rotman, An introduction to homological algebra, Academic Press, London and New York, 1979.

11. S. P. Smith, An example of a ring Morita equivalent to the Weyl algebra $A_{1}$, J. Algebra 73 (1981), 552-555.

12. S. P. Smith and J. T. Stafford, Differential operators on an affine curve (to appear).

13. J. T. Stafford, On the stable range of right Noetherian rings, Bull. London Math. Soc. 13 (1981), 39-41.

14. _ Stably free, projective right ideals, Compositio Math. 54 (1985), 63-78.

15. R. G. Swan, Vector bundles and projective modules, Trans. Amer. Math. Soc. 105 (1962), 264-277.

16. W. Van der Kulk, On polynomial rings in two variables, Nieuw Arch. Wisk. (3) 1 (1953), 33-41.

17. D. B. Webber, Ideals and modules of simple Noetherian hereditary rings, J. Algebra 16 (1970), 239-242.

Department of Mathematics, Leeds University, Leeds LS2 9JT, England (Current address)

Department of Mathematics, University of Washington, Seattle, WashingTON 98195 